\title{
Evaluation of Self-Heating and Hot Carrier Degradation of Poly-Si Thin-Film Transistors using Charge Pumping technique
}

\author{
Xiaowei LU, *Mingxiang WANG, Kai SUN, and Lei LU \\ Dept. of Microelectronics, Soochow University, No.1 Shizi Street, Suzhou, 215006, China \\ *Email: Mingxiang_wang@suda.edu.cn
}

\begin{abstract}
Self-heating (SH) and hot carrier (HC) degradation of n-type poly-Si thin-film transistors (TFTs) is evaluated by using charge pumping (CP) technique. By extracting trap state energy distribution, it is demonstrated that $\mathrm{SH}$ degradation is mainly attributed to the generation of deep states. For HC stressed TFTs, an anomalous $I_{C P}$ decrease with the stress time is observed in a low $V_{g}$ stress condition controlled by hole trapping; while in a mid $V_{g}$ condition, CP signal clearly indicates the trap states generation controlled by electron trapping.
\end{abstract}

Keywords: charge pumping, poly-Si TFTs, self-heating, hot carrier

\section{INTRODUCTION}

Charge pumping (CP) is a technique providing direct information on interface trap properties [1], and has been extensively employed to investigate device degradation in MOSFETs [2]. While in poly-Si thin-film transistors (TFTs), there are very limited applications of CP for hot carrier (HC) or NBTI degradation [3, 4]. Recently, we showed that measurement optimization was critical to achieve reliable $\mathrm{CP}$ characterization in poly-Si TFTs [5]. In this work, such optimized CP technique is employed to evaluate their selfheating $(\mathrm{SH})$ and $\mathrm{HC}$ degradation. For $\mathrm{SH}$ degradation, trap states generation mainly on the deep states is observed. While for HC degradation, different CP characteristics are observed in low and mid $V_{g}$ stress conditions. An abnormal $I_{C P}$ decrease with the stress time is observed in low $V_{g}$ stressed TFTs, which is different from previous observations [3], whereas in mid $V_{g}$ stressed TFTs, CP characterization clearly indicates the electron trapping dominated trap states generation.

\section{EXPERIMENTAL}

$\mathrm{N}$-type TFT with a $\mathrm{p}^{+}$side contact connecting the poly-Si body is used in this study. As shown in Fig.1a is a planar view of the device under test. The $100 \mathrm{~nm}$ poly-Si active layer was formed by solution based metal-induced crystallization of a-Si. Wafers were first dipped in nickel nitrate solution, and then crystallized at $630{ }^{\circ} \mathrm{C}$ in $\mathrm{N}_{2}$ ambient. Subsequently the poly-Si film was recrystallized at $900{ }^{\circ} \mathrm{C}$. Gate oxide was formed by polyoxidation at $950{ }^{\circ} \mathrm{C}$ in dry $\mathrm{O}_{2}$ for 48 mins. Phosphorous implantation was introduced to form the source and drain with a dose of $4 \times 10^{15} \mathrm{~cm}^{-2}$, then followed by a dopant activation at $900{ }^{\circ} \mathrm{C}$ for $1.5 \mathrm{~h}$. Device $\mathrm{W} / \mathrm{L}$ for $\mathrm{SH}$ stress test is $15 / 4 \mu \mathrm{m}$ while for $\mathrm{HC}$ stress is $10 / 4$ or $20 / 4 \mu \mathrm{m}$. For SH stress,
$V_{g} / V_{d}=12 / 14 \mathrm{~V}$ is applied with a stress power about $47 \mathrm{~mW}$. For HC stress, low $V_{g}$ and mid $V_{g}$ stress are applied with bias stress of $V_{g} / V_{d}=2 / 10 \mathrm{~V}$ and $6 / 12 \mathrm{~V}$, respectively.

Fig. $1 \mathrm{~b}$ is a cross sectional view of CP measurement. All stress test and CP measurement are performed at room temperature. Before and after a $\mathrm{HC}$ or $\mathrm{SH}$ stress, the TFT is pulsed from accumulation to inversion using a square $V_{g}$ pulse with the source (S) and drain (D) grounded. By adjusting the pulse base voltage $\left(V_{g b}\right)$ while keeping a constant pulse height $\left(V_{p h}\right)$, an Elliot curve is measured from the $\mathrm{p}^{+}$side contact $[1,5]$ for $\mathrm{CP}$ analysis. In the $\mathrm{CP}$ measurement, the pulse period $\left(T_{p}\right)$ is $100 \mu \mathrm{s}$ and $V_{p h}$ is fixed at $5 \mathrm{~V}$. The pulse rising and falling time $\left(T_{r}, T_{f}\right)$ are chosen as $5 \mu$ s, long enough to minimize the geometric effect [5]. Thus reliable and well shaped CP curves can be obtained. Besides, transfer curves are also measured before and after the degradation by using Agilent $4156 \mathrm{C}$ analyzer and Vector MX-1100B prober.

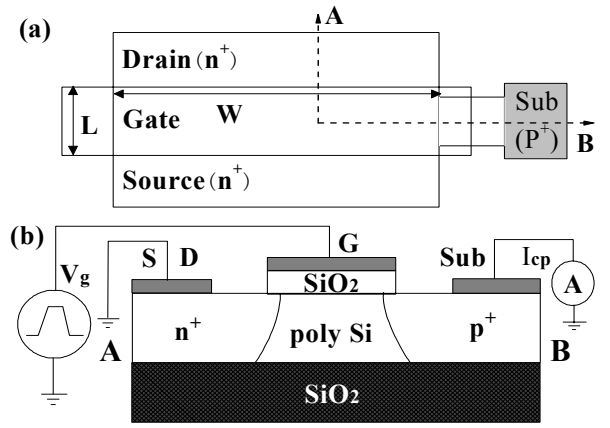

Figure 1. (a). Planar view of the high temperature processed TFT under test. (b). Cross sectional view of $\mathrm{CP}$ measurement.

\section{RESULTS AND DISCUSSIONS}

\section{SH Degradation}

Shown in Fig. 2 is typical transfer curve degradation of a $\mathrm{SH}$ stressed TFT [6]. The subthreshold slope degrades slightly, resulting in a positive shift of device $V_{t h}$. On current $\left(I_{o n}\right)$ decreases while leakage current $\left(I_{\text {off }}\right)$ increases. In Fig.3 the Elliot curves measured at different stress times are shown. Clearly, CP current $\left(I_{C P}\right)$ significantly increases with stress time, indicating the generation of interface traps. However, almost no shift in the Elliot curves is observed at either transition edge. Thus there should be no net charge generated in the gate oxide during the stress. 


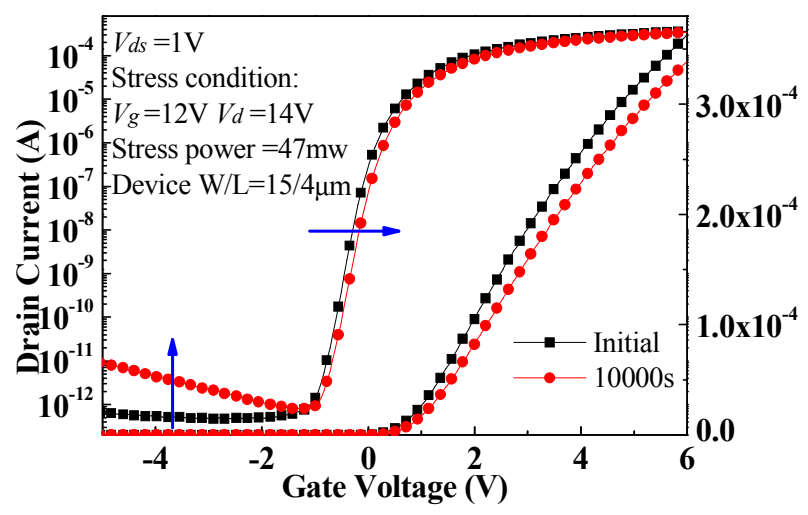

Figure 2. Linear and logarithmic plots of the typical transfer curves under SH stress, $V_{g} / V_{d}=12 / 14 \mathrm{~V}$, with a stress power of about $47 \mathrm{mw}$.

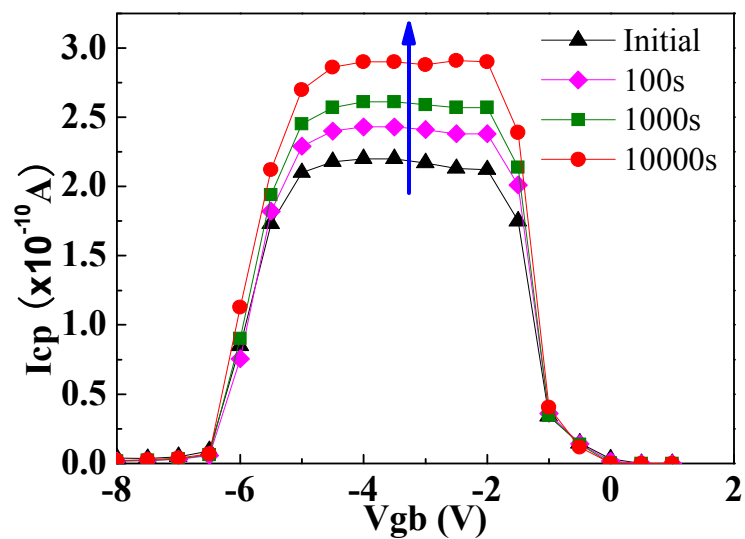

Figure 3. Charge pumping characteristics as a fucntion of pulse base voltage at various stress times. Distinct increase of $I_{C P}$ is observed.

In Fig. 4 inset, the maximum $I_{C P}$ linearly increases with the measurement pulse frequency $f$, from which average trap densities $\left(D_{t}\right)$ are extracted for all stress times using the equation [1] as below:

$$
I_{c p}=2 D_{t}\left[\ln \left(V_{t h n i} \sqrt{\sigma_{n} \sigma_{p}}\right)+\ln \left(\frac{\left|V_{f b}-V_{t}\right|}{V_{p h}} \sqrt{T_{r} T_{f}}\right)\right] k T A_{g q f}
$$

The capture cross section $\left(\sigma_{n} \sigma_{p}\right)^{1 / 2}$ is set as $2.4 \times 10^{-16} \mathrm{~cm}^{2}$ [5]. Shown in Fig.4 is the extracted $D_{t}$ as a function of stress time. In Fig.5 normalized degradation curves for key device parameters as well as $D_{t}$ are compared. All curves follow a power law dependence on the stress time with about the same time exponent of 0.21 , showing the correlation between the device degradation and trap states generation.

Furthermore, by measuring $I_{C P}$ with fixed $T_{r}$ and variable $T_{f}$, trap energy distribution $D_{t}(E)$ within the upper half of the band gap for each stress time can be extracted using the equation [1]:

$$
D_{t}(E)=-\frac{T_{f}}{q f A g k T} \frac{d I_{c p}}{d T_{f}}
$$

In Fig. 6 both deep states and tail states are seen to steadily increase with stress time, however, larger increase is observed near the mid-gap than that near the band edge. Deep states are normally attributed to dangling bonds due to bond breaking, while tail states to strained bonds [6]. Thus the observation here is a direct evidence to the SH trap generation mechanism related to $\mathrm{Si}-\mathrm{H}$ or $\mathrm{Si}-\mathrm{Si}$ breaking at the $\mathrm{Si}-\mathrm{SiO}_{2}$ interface or grain boundaries. In previous studies $[7,8]$, the same mechanism was proposed based on simulation or indirect observations, to which such direct $\mathrm{CP}$ evidence can be a better support. On the other hand, it also demonstrates the feasibility of the $\mathrm{CP}$ technique in probing the trap states generation in $\mathrm{SH}$ degraded poly-Si TFTs.

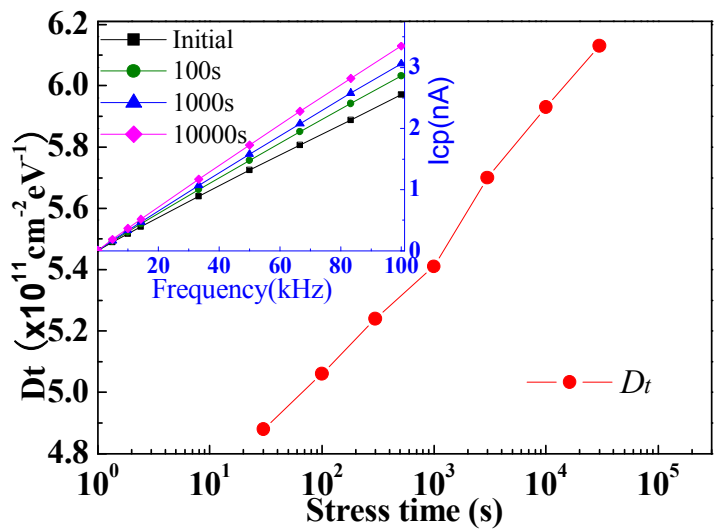

Figure 4. Stress time dependence of trap state density $\left(D_{t}\right)$. The inset is frequency dependence of CP current, where average $D_{t}$ can be extracted by the given equation (1).

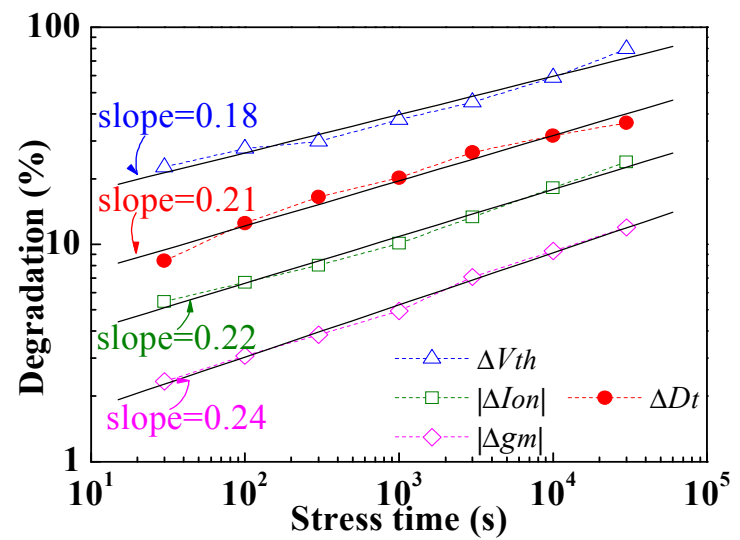

Figure 5. Stress time dependence of key device parameters degradation. $D_{t}$ and device parameters follow a power law with about the same time exponent of 0.21 .

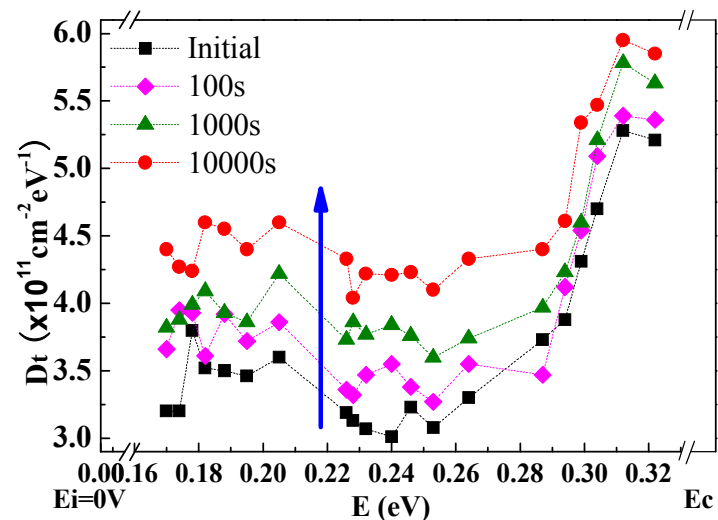

Figure 6. Trap state energy distribution $D_{t}(E)$ is extracted by keeping pulse $T_{r}$ constant and varying $T_{f}$. SH degradation is mainly attributed to the generation of deep states. 


\section{HC Degradation}

Shown in Fig.7 is transfer curve degradation of a low $V_{g}$ HC stressed TFT. The subthreshold region continuously shifts to the negative and degrades severely. $I_{\text {off }}$ increases distinctly, whereas negligible change occurs in $I_{o n}$. Similar transfer curve degradation was also seen in other high temperature processed TFTs under similar low $V_{g} \mathrm{HC}$ stress condition [9]. Shown in Fig. 8 are $\mathrm{CP}$ curves similarly measured by optimized CP technique as in SH degradation. The right edge of the Elliot curve clearly shifts to the negative, in accordance with the negative shift of the subthreshold region in Fig.7, indicating the generation of positive charges in the gate oxide [2]. Indeed, hot hole injection into the gate oxide is favored under a low $V_{g} \mathrm{HC}$ stress condition [2]. Surprisingly, $I_{C P}$ clearly decreases with the stress times. It's a different observation from previous $\mathrm{CP}$ result also in low $V_{g}$ HC stressed TFTs [3]. In order to verify such observation, pulse $T_{r}$ and $T_{f}$ is varied from 100 ns to 500 $\mu \mathrm{s}$, all measured $I_{C P}$ similarly decreases, eliminating the possibility of $\mathrm{CP}$ measurement error.

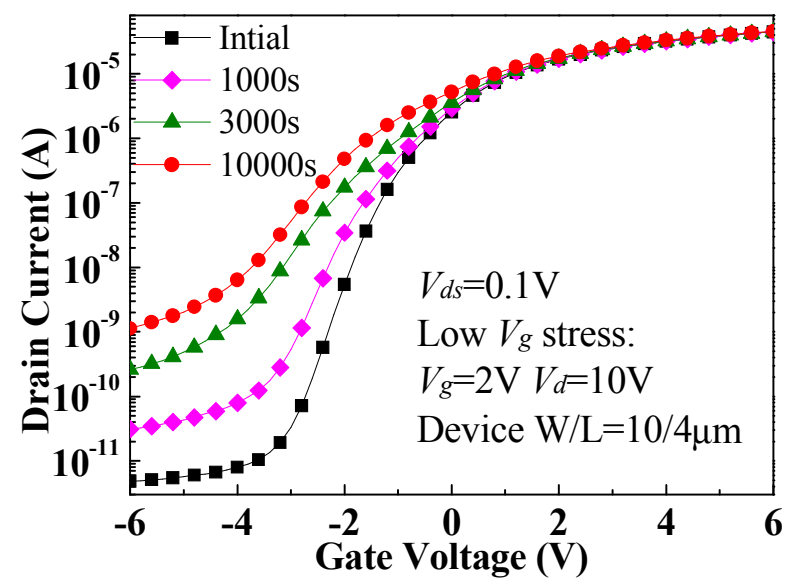

Figure 7. Stress time dependence of transfer curves under low $V_{g} \mathrm{HC}$ stress, $V_{g} / V_{d}=2 / 10 \mathrm{~V}$. Device $\mathrm{W} / \mathrm{L}=10 / 4 \mu \mathrm{m}$.

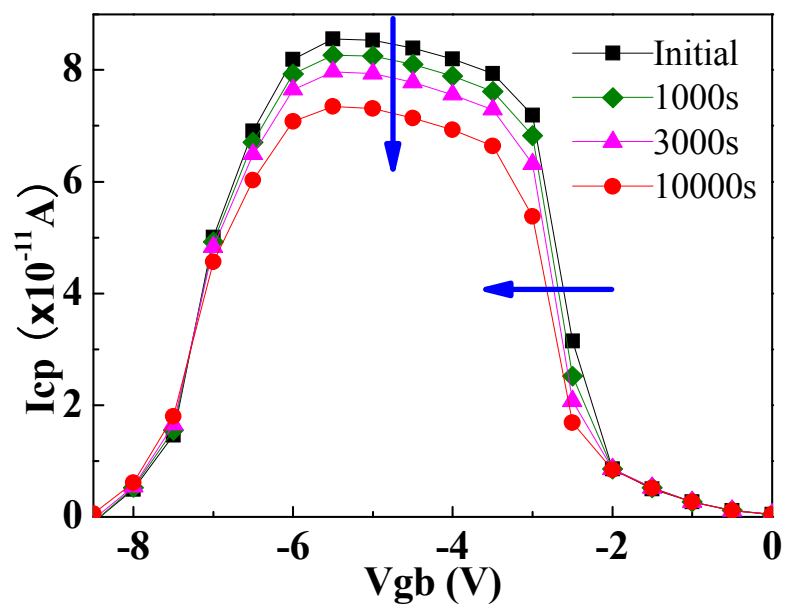

Figure 8. Charge pumping current versus pulse base voltage of the device under a low $V_{g} \mathrm{HC}$ stress.

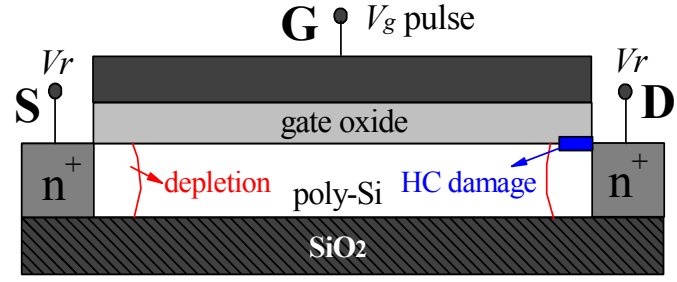

Figure 9. Schematic cross-sectional view of device in CP measurement. HC induced damage region can be masked by drain depletion region due to applied reverse bias $V_{r}$.

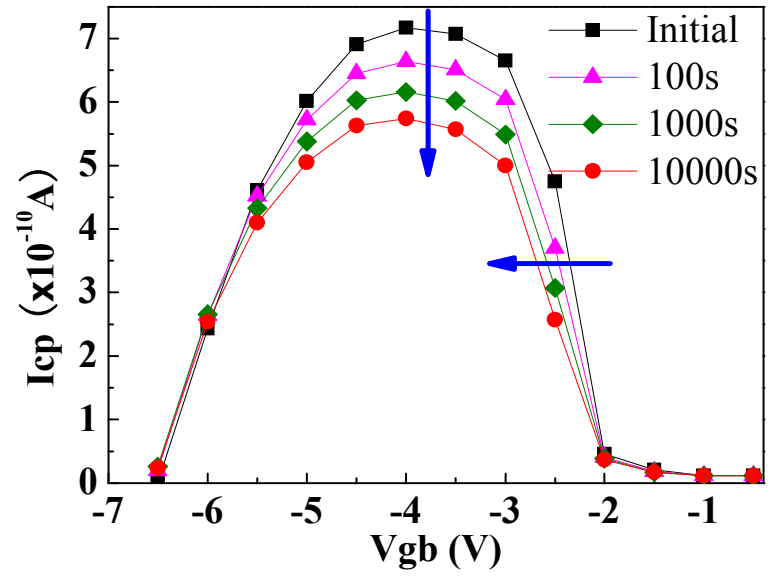

Figure 10. Charge pumping characteristics of device under a low $V_{g} \mathrm{HC}$ stress measured with the source and drain reversely biased at $V_{r}=1 \mathrm{~V}$.

Obviously, a conclusion of interface trap reduction based on the observed $I_{C P}$ decrease can not be true. Thus one may consider that the effective channel length shortening due to $\mathrm{HC}$ injection into the gate oxide [2] may cause an $I_{C P}$ reduction. To clarify this point, $\mathrm{CP}$ curve is measured with the source and drain reversely biased at $V_{r}=1 \mathrm{~V}$, as shown in Fig.9. In such condition, $\mathrm{HC}$ induced damaged region [6] or $\mathrm{HC}$ injection induced local inversion region [2] at the drain end can be masked by a wider drain depletion region. Therefore, only the central channel region is probed by $\mathrm{CP}$. As expected, such measured $I_{C P}$ is reduced compared to that with $\mathrm{S} / \mathrm{D}$ grounded, which is attributed to the reduction of effective gate area $\left(A_{g}\right)$ as given in Eq.(1). However, the continuous $I_{C P}$ decrease with the stress time still remains as shown in Fig.10. Thus we tend to believe that conventional $I_{C P}$ measurement is not sensitive to the HC trap states generation dominated by hole trapping mechanism [10].

In a mid $V_{g} \mathrm{HC}$ stress condition, interface traps are generated through both electron and hole trapping [2]. Fig.11 is transfer curve degradation of a TFT stressed at $V_{g} / V_{d}=6 / 12 \mathrm{~V}$. In the inset, a positive shift of the right edge of the Elliot curve indicates the generation of net negative charges due to more electron trapping. Interestingly, here $I_{C P}$ clearly increases with stress time, in agreement with the $\mathrm{HC}$ induced trap generation. Therefore, in both cases of HC degradation of poly-TFTs, shift of CP Elliot curve is a good indication of the respective trap generation mechanism, while one should be tentative when quantitatively evaluating trap generation using the $I_{C P}$ variation. However, further investigation is needed to clarify the origin of the anomalous $\mathrm{CP}$ decrease under a low $V_{g} \mathrm{HC}$ stress. 


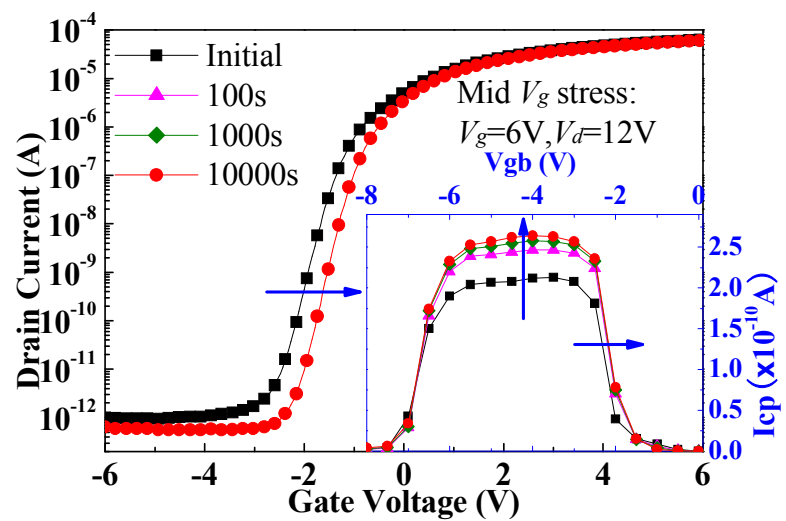

Figure 11. Transfer curve degradation of device under a mid $V_{g} \mathrm{HC}$ stress, $V_{g} / V_{d}=6 / 12 \mathrm{~V}$. Inset is degradation of $\mathrm{CP}$ characteristics, indicating the electron trapping dominated trap states generation. Device $\mathrm{W} / \mathrm{L}=20 / 4 \mu \mathrm{m}$.

\section{CONCLUSION}

The optimized CP technique was employed to evaluate the self-heating and hot-carrier degradation in poly-Si TFTs. SH degradation is mainly attributed to the generation of deep states. For HC degradation, $\mathrm{CP}$ exhibits different behaviors associated with different trap generation mechanisms. In the low $V_{g}$ stress condition, the anomalous $I_{C P}$ decrease is in contradiction to the HC trap states generation. While for the mid $V_{g} \mathrm{HC}$ stress induced degradation, $\mathrm{CP}$ characterization clearly indicates the electron trapping dominated trap states generation.

\section{REFERENCES}

[1] G. Groeseneken, H. E. Maes, N. Beltran, and R. F. De Keersmaecker, "A reliable approach to charge-pumping measurements in MOS transistors," IEEE Trans. Electron Devices, vol ED-31, NO. 1, pp. 42-53, Jan. 1984.
[2] Paul Heremans, Rudi Bellens, Guido Groeseneken, and Herman E. Maes, "Consistent Model for the Hot-Carrier Degradation in n-Channel and pChannel MOSFET's," IEEE Trans. Electron Devices, vol. 35, NO. 12, December, 1988.

[3] T. Yoshida, K. Yoshino, M. Takei, A. Hara, N. Sasaki, and T. Tsuchiya "Experimental Evidence of Grain-Boundary Related Hot-Carrier Degradation Mechanism in Low-Temperature Poly-Si Thin-FilmTransistors," IEEE IEDM, 2003.

[4] Chih-Yang Chen, Ming-Wen Ma, Wei-Cheng Chen, Hsiao-Yi Lin, Kuan-Lin Yeh, Shen-De Wang, and Tan-Fu Lei, "Analysis of Negative Bias Temperature Instability in Body-Tied Low-Tempreature Polycrystalline Silicon Thin-Film Transistors," IEEE Electron Device Letters, vol. 29, NO. 2, February 2008.

[5] Lei Lu, Mingxiang Wang, and Man Wong, "Geometric Effect Elimination and Reliable Trap State Density Extraction in Charge Pumping of Polysilicon Thin-Film Transistors," IEEE Electron Device Letters, vol. 30, NO. 5, May 2009.

[6] N.A. Hatas, A. Archontas, C.A. Dimitriadis, G. Kamarinos, T. Nikolaidis, N. Georgoulas, and A. Thanailakis, "Substrate current and degradation of n-channel polycrystalline silicon thin film transistors," Microelectronics Reliability, vol 45, 2005, pp. 341-348.

[7] Huaisheng Wang, Mingxiang Wang, Zhenyu Yang, Han Hao, and Man Wong, "Stress Power Dependent Self-Heating Degradation of MetalInduced Laterally Crystallized n-Type Polycrystalline Silicon Thin-Film Transistors," IEEE Trans. Electron Devices, vol.54, NO. 12, December 2007.

[8] Satoshi Inoue, Hiroyuki Ohshima, and Tatsuya Shimoda, "Analysis of Degradation Phenomenom Caused by Self-Heating in LowTemperature-Processed Polycrystalline Silicon Thin Film Transistors," Jpn. J. Appl. Phys. Vol. 41, 2002, pp. 6313-6319.

[9] F.V. Farmakis, C.A. Dimitriadis, J. Brini, G. Kamarinos, V. K. Gueorguiev and Tz. E. Ivanov, "Hot-carrier phenomena in high temperature processed undoped-hydrogenated n-channel polysilicon thin film transistors," Solid-State Electronics (1999) 1259-1266.

[10] D. J. DiMaria, D. A. Buchanan, J. H. Stathis, and R. E. Stahlbush, "Interface states induced by the presence of trapped holes near the silicon-silicon-dioxide interface," Journal of Applied Physics, vol 77, 1995, pp. 2032-2040. 